\title{
Mevalonate Pathway Promiscuity Enables Noncanonical Terpene Production
}

Eiben, Christopher B.; De Rond, Tristan; Bloszies, Clayton; Gin, Jennifer; Chiniquy, Jennifer; Baidoo, Edward E.K.; Petzold, Christopher J.; Hillson, Nathan J.; Fiehn, Oliver; Keasling, Jay D.

Published in:

ACS Synthetic Biology

Link to article, DOI:

10.1021/acssynbio.9b00230

Publication date:

2019

Document Version

Early version, also known as pre-print

Link back to DTU Orbit

Citation (APA):

Eiben, C. B., De Rond, T., Bloszies, C., Gin, J., Chiniquy, J., Baidoo, E. E. K., Petzold, C. J., Hillson, N. J., Fiehn, O., \& Keasling, J. D. (2019). Mevalonate Pathway Promiscuity Enables Noncanonical Terpene Production. ACS Synthetic Biology, 8(10), 2238-2247. https://doi.org/10.1021/acssynbio.9b00230 


\title{
Mevalonate Pathway Promiscuity Enables Non-Canonical Terpene Production
}

Author List:

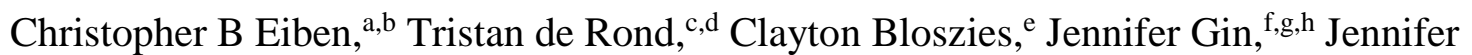
Chiniquy, ${ }^{\text {f,g,h }}$ Edward EK Baidoo, ${ }^{\text {f,g,h }}$ Christopher J Petzold, ${ }^{\text {f,g,h }}$ Nathan J Hillson, ${ }^{\text {f,g,h }}$ Oliver Fiehn, ${ }^{\mathrm{e}}$ Jay D Keasling*,a,f,g,i,j,k,l

${ }^{a}$ Department of Bioengineering, ${ }^{c}$ Department of Chemistry, ${ }^{i}$ Department of Chemical and Biomolecular Engineering, and ${ }^{\mathrm{j} I n s t i t u t e}$ for Quantitative Biosciences, University of California, Berkeley, CA 94270

${ }^{b}$ Department of Bioengineering, University of California, San Francisco, CA 94143

${ }^{\mathrm{d}}$ Center for Marine Biotechnology and Biomedicine, Scripps Institution of Oceanography, University of California San Diego, La Jolla, CA 92093

eNational Institute of Health West Coast Metabolomics Center, University of California Davis, Davis, CA 95616

${ }^{\mathrm{f}}$ Department of Energy Joint BioEnergy Institute, 5885 Hollis Street, Emeryville, CA 94608

'Biological Systems and Engineering Division, Lawrence Berkeley National Laboratory, Berkeley, CA 94720

hDepartment of Energy Agile BioFoundry, Emeryville, CA, 94608

${ }^{k}$ Novo Nordisk Foundation Center for Biosustainability, Technical University of Denmark, Hørsholm, Denmark

${ }^{1}$ Center for Synthetic Biochemistry, Institute for Synthetic Biology, Shenzhen Institutes for Advanced Technologies, Shenzhen, China

*To whom correspondence may be addressed. Email: JDKeasling@lbl.gov

\begin{abstract}
:
Lepidoptera (butterflies and moths) make the six-carbon compounds homoisopentenyl pyrophosphate (HIPP) and homodimethylallyl pyrophosphate (HDMAPP) that are incorporated into sixteen, seventeen and eighteen carbon farnesyl pyrophosphate (FPP) analogues. In this work we heterologously expressed the lepidopteran modified mevalonate pathway, a propionylCoA ligase, and terpene cyclases in $E$. coli to produce several novel terpenes containing sixteen carbons. Changing the terpene cyclase generated different novel terpene product profiles. To further validate the new compounds we confirmed ${ }^{13} \mathrm{C}$ propionate was incorporated, and that the masses and fragmentation patterns were consistent with novel sixteen carbon terpenes by GCQTOF. Based on the available farnesyl pyrophosphate analogues lepidoptera produce, this approach should greatly expand the reachable biochemical space with applications in areas where terpenes have traditionally found uses.
\end{abstract}

\section{Keywords:}


Mevalonate Pathway, Terpenes, Juvenile Hormones, homoisopentenyl pyrophosphate, HIPP, homodimethylallyl pyrophosphate, HDMAPP

With an estimated 55,000 chemical species, terpenoids are the largest natural products class ${ }^{1}$. Terpenoids fulfill many important biological roles including cell wall components (sterols) ${ }^{2}$, quinones in the electron transport chain ${ }^{3}$, protein trafficking/localization regulators (prenylation) ${ }^{4}$, signaling molecules (estrogen $)^{5}$, and bioactive molecules for interspecies competition ${ }^{6}$. In addition terpenoids have important economic uses as pharmaceuticals (taxol) ${ }^{7}$, flavors (linalool) $)^{8}$, fragrances (patchoulol) $)^{9}$, commodity chemicals (isoprene, limonene) $)^{10,11}$ and biofuels (bisabolane and isopentanol) ${ }^{12,13,14}$.

Despite their diversity, terpenoids canonically derive from isopentenyl pyrophosphate (IPP) and dimethylallyl pyrophosphate (DMAPP), interconvertible five-carbon metabolites which can combine forming longer prenyl pyrophosphates (Fig. 1A). Each IPP ligation yields a molecule five carbons larger, giving the progression of geranyl (ten carbons), farnesyl (fifteen carbons), geranylgeranyl (twenty carbons) pyrophosphates, and so on. Terpene synthases can catalyze the conversion of prenyl pyrophosphates into diverse terpene scaffolds via carbocation rearrangements and cyclizations, producing a stunning molecular diversity further magnified by downstream tailoring enzymes. Despite the tailoring, most terpene backbones retain the characteristic five, ten, fifteen, etc. carbons.

When necessary, nature uses several strategies to produce terpene backbones outside the multiple of five carbon sequence. The most common strategies are carbon removal from larger terpene backbones, as in geosmin biosynthesis ${ }^{15}$, or attaching another carbon containing molecule, as in meroterpenoid biosynthesis such as the bitter acids in Humulus lupulus $L$. (hops) ${ }^{16}$. A comparatively less common strategy observed in nature uses methyltransferases, which add methyl groups from S-adenosyl methionine (SAM) to prenyl pyrophosphate molecules as in 2-methylisoborneol ${ }^{17}$ or longestin ${ }^{18}$ biosynthesis. Recently this SAMmethyltransferase strategy has been developed to produce novel terpenes. The strategies differ mostly by methyl incorporation location, and the prenyl pyrophosphate used as methyltransferase substrate, with successful examples demonstrated for IPP ${ }^{19}$, GPP $^{20,21}$ and FPP ${ }^{22}$.

Lepidoptera employ an alternate strategy to create endocrine molecules named Juvenile Hormones (JHs) containing terpene backbones of sixteen, seventeen, and eighteen carbons (Fig. 1D). Lepidoptera incorporate the six-carbon building blocks homo-IPP (HIPP) and homoDMAPP (HDMAPP) into $\mathrm{JHs}^{23}$ through a modified mevalonate pathway. The first lepidopteran mevalonate pathway step condenses propionyl-CoA and acetyl-CoA (Fig. 1B), instead of the canonical two acetyl-CoAs (Fig. 1A). The extra methyl from propionyl-CoA is retained through the lepidopteran mevalonate pathway forming FPP analogues with sixteen, seventeen, and eighteen carbons en route to JH biosynthesis (Fig. 1B).

Several qualities make the lepidopteran mevalonate pathway attractive for exploring novel terpene space that synthetic chemistry struggles to efficiently sample (potential examples Fig. 1C). Relatively few FPP analogues can theoretically produce significant chemical diversity 
by varying a single enzyme, the terpene cyclase. In previous work terpene cyclases have successfully cyclized chemically synthesized prenyl pyrophosphate analogues similar to the lepidopteran FPP analogues ${ }^{24}$. To our knowledge, only three non-JH terpenes likely incorporate propionate in nature. Though their full biosynthetic pathways remain unknown, 3-methyl- $\alpha$ himachalene, a Lutzomyia longipalpis (sandfly) sex pheromone ${ }^{25}$ has sixteen carbons, and homofarnesene and bishomofarnesene, Myrmica Ants trail pheromones ${ }^{26}$, have sixteen and seventeen carbons, respectively. For these technical reasons, and the potential medicinal and industrial uses novel terpenes offer, we investigated novel terpene production in E. coli using the lepidopteran mevalonate pathway paired with various terpene cyclases.

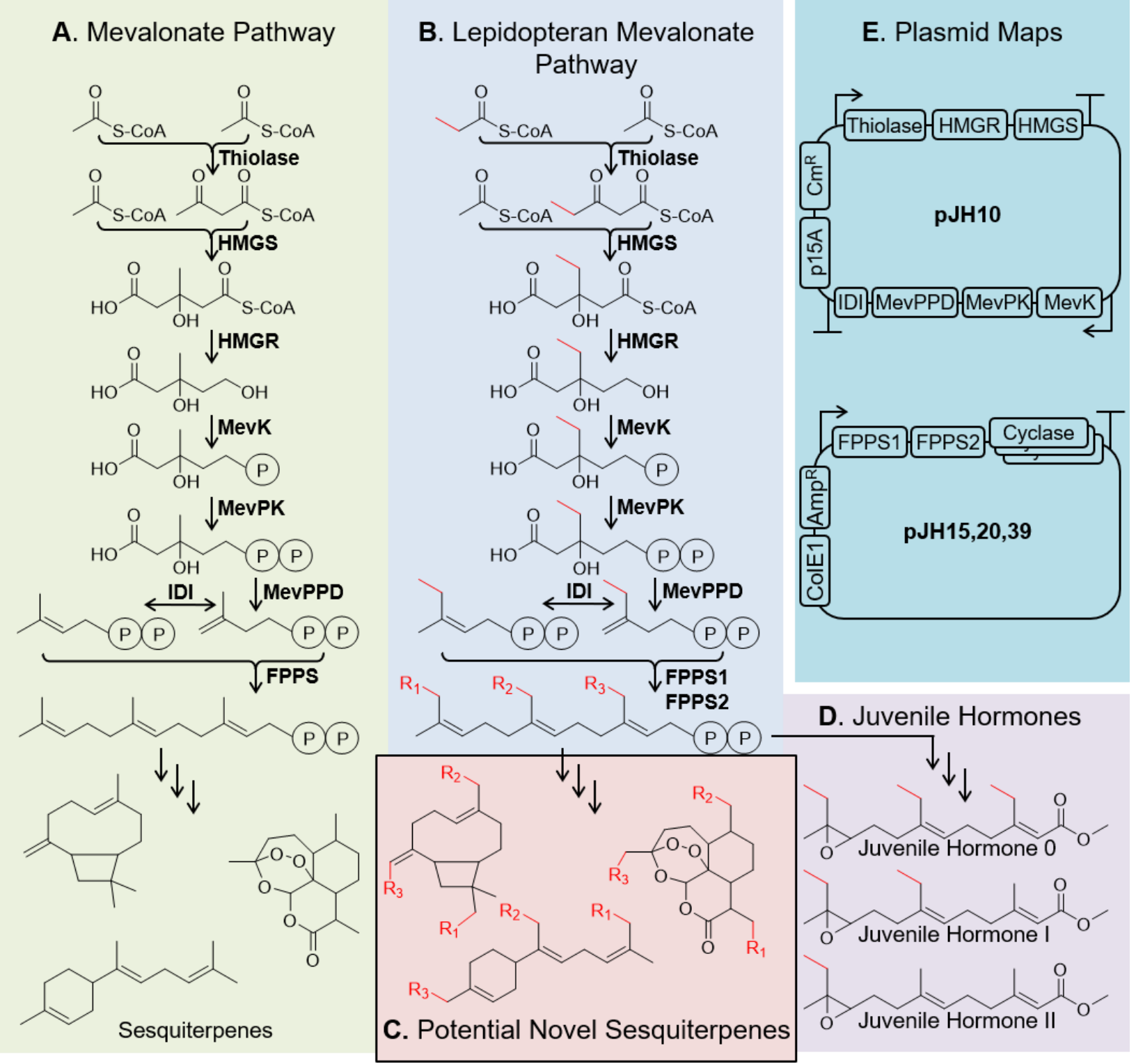

Fig. 1. Overview of the canonical mevalonate pathway, the lepidopteran mevalonate pathway, chemical diversity of juvenile hormones and plasmid architecture of pJH10, pJH15, pJH20, and pJH39. A. The canonical mevalonate pathway. The pathway starts with the condensation of two acetyl-CoA and ultimately produces FPP, an essential metabolite. B. The lepidopteran 
mevalonate pathway. Production of FPP analogues for juvenile hormone biosynthesis begins with the condensation of a propionyl-CoA and acetyl-CoA instead of the usual two acetyl-CoA. The extra methyl group is shown in red for emphasis. The core enzymes of the lepidopteran mevalonate pathway also produce FPP via the canonical reactions. C. Examples of potential sesquiterpenes that could be derived from the lepidopteran FPP analogues. D. Examples of several lepidopteran Juvenile Hormones used as endocrine molecules showcasing some of the different locations the extra methyl can incorporate. E. Plasmid maps of pJH10, pJH15, pJH20, and pJH39. pJH15, pJH20 and pJH39 only differ in terpene cyclase. Enzyme abbreviations: [HMGS: 3-hydroxy-3-methylglutaryl-coenzyme A synthase], [HMGR: 3-hydroxy-3methylglutaryl-coenzyme A reductase], [MevK: mevalonate kinase], [MevPK: mevalonate phosphate kinase], [MevPPD: mevalonate pyrophosphate decarboxylase], [IDI: isopentenyl diphosphate delta isomerase], [FPPS: farnesyl pyrophosphate synthase]. $\left[\mathrm{R}_{1}=\mathrm{H}, \mathrm{Me}\right],\left[\mathrm{R}_{2}=\mathrm{H}\right.$, $\mathrm{Me}],\left[\mathrm{R}_{3}=\mathrm{H}, \mathrm{Me}\right]$

\section{Results and Discussion:}

Lepidopteran mevalonate pathway enzyme bioprospecting and plasmid design. Lepidoptera have only one apparent enzyme for each mevalonate pathway step except the thiolase and FPP synthase steps ${ }^{27}$. The two FPP synthases from the lepidoptera Choristoneura fumiferana (eastern spruce budworm) have been previously expressed recombinantly and purified from $E$. coli, thus we selected them ${ }^{28}$. It was noted that combining purified cfFPPS1 and cfFPPS2, thought to form a heterodimer, increased FPP analogue production in previous work ${ }^{28}$ so our designs express both enzymes concurrently. For the remaining mevalonate pathway enzymes we selected those predicted by Kinjoh et al. from the lepidopteran Bombyx mori (silkworm) ${ }^{27}$. Finally we selected the well-characterized terpene cyclase epi-isozizaene synthase ${ }^{30}$.

The predicted Bombyx mori thiolase did not express in E. coli, so we used the wellcharacterized thiolase PhaA from Acinetobacter strain RA384931. Soluble expression of the Bombyx mori 3-hydroxy-3-methylglutaryl-coenzyme A (bmHMGR) required truncating the membrane-associated N-terminal domain, similarly required for the $S$. cerevisiae HMGR homologue over expression in other work ${ }^{32}$.

We cloned the genes into two plasmids named pJH10 and pJH15 based on plasmids pBbA5c-MevT(CO)-T1-MBIS(CO, ispA) ${ }^{12}$ and pBbE1a-GPPS-LS ${ }^{11}$, respectively. Annotated genbank files for the plasmid are supplied on the JBEI ICE open registry (see Supplementary Information $)^{33}$. Briefly, pJH10 expresses the first mevalonate pathway section, from thiolase to isopentenyl-pyrophosphate isomerase, on a medium-copy plasmid (Fig. 1E). pJH15 has the two FPP synthases and epi-isozizaene synthase driven by a strong promoter on a high copy plasmid (Fig. 1E).

Heterologous pathway expression in $\boldsymbol{E}$. coli and small molecule analysis. We used the E. coli expression strain Bap1 because it lacks propionate catabolism and has a strong T7 inducible promoter driving the genomic propionyl-CoA synthetase $(p r p E)^{34}$. We grew E. coli Bap1 cells 
harboring both pJH10 and pJH15 plasmids in sodium propionate supplemented Terrific Broth medium. At induction we reduced the temperature to $18^{\circ} \mathrm{C}$ to aid correct folding of the lepidopteran enzymes and added a nonane overlay to continuously extract terpenes into the hydrophobic layer. After 36 hours we harvested cell pellets for proteomic analysis and the overlay for Gas Chromatography - Mass Spectrometry (GC-MS) small molecule analysis. Proteomics confirmed protein expression, finding all pJH10 and pJH15 proteins expressed in $E$. coli Bap1 cells (Fig. S2-5) above the no plasmid control. In the nonane overlay several new peaks with $\mathrm{m} / \mathrm{z}=218$ molecular ions were observed, consistent with novel $\mathrm{C}_{16} \mathrm{H}_{26}$ terpenes (Fig. 2A). Replacing pJH15 with the control plasmid pBbE1a-RFP ${ }^{35}$, which encodes red fluorescent protein rather than the FPP synthases and a terpene cyclase, abolished the new peaks, as did sodium propionate omission. When feeding ${ }^{13} \mathrm{C}$ propionate, singly-labeled at the carboxylic acid position, a corresponding $+1 \mathrm{~m} / \mathrm{z}$ parent ion mass shift (Fig. 2A) was seen in the novel compounds via GC-MS.

We then tested if alternate terpene cyclases could also produce $\mathrm{C}_{16} \mathrm{H}_{26}$ terpenes. We built additional plasmids pJH20 and pJH39 based on pJH15, replacing epi-isozizaene synthase with, respectively, the alternate terpene cyclases $\alpha$-humulene synthase from Zingiber zerumbet (bitter ginger) ${ }^{36}$ and $\gamma$-humulene synthase mutant S484A, Y566 $\mathrm{F}^{37}$ from Abies grandis (grand fir). The $\gamma$-humulene synthase mutant was expressed as a maltose binding protein (MBP) fusion to aid solubility (Fig. 1E). These terpene cyclases also produced novel peaks compared to the RFP control (Fig. 2B, 2C), with each synthase producing a distinct product profile. All the novel peaks elute off the GC column after the sesquiterpene $\beta$-caryophyllene, consistent with the novel peaks having a higher molecular weight than this fifteen-carbon standard. Although theoretically possible, we could not confirm novel peaks consistent with seventeen or eighteen carbon terpenes. 

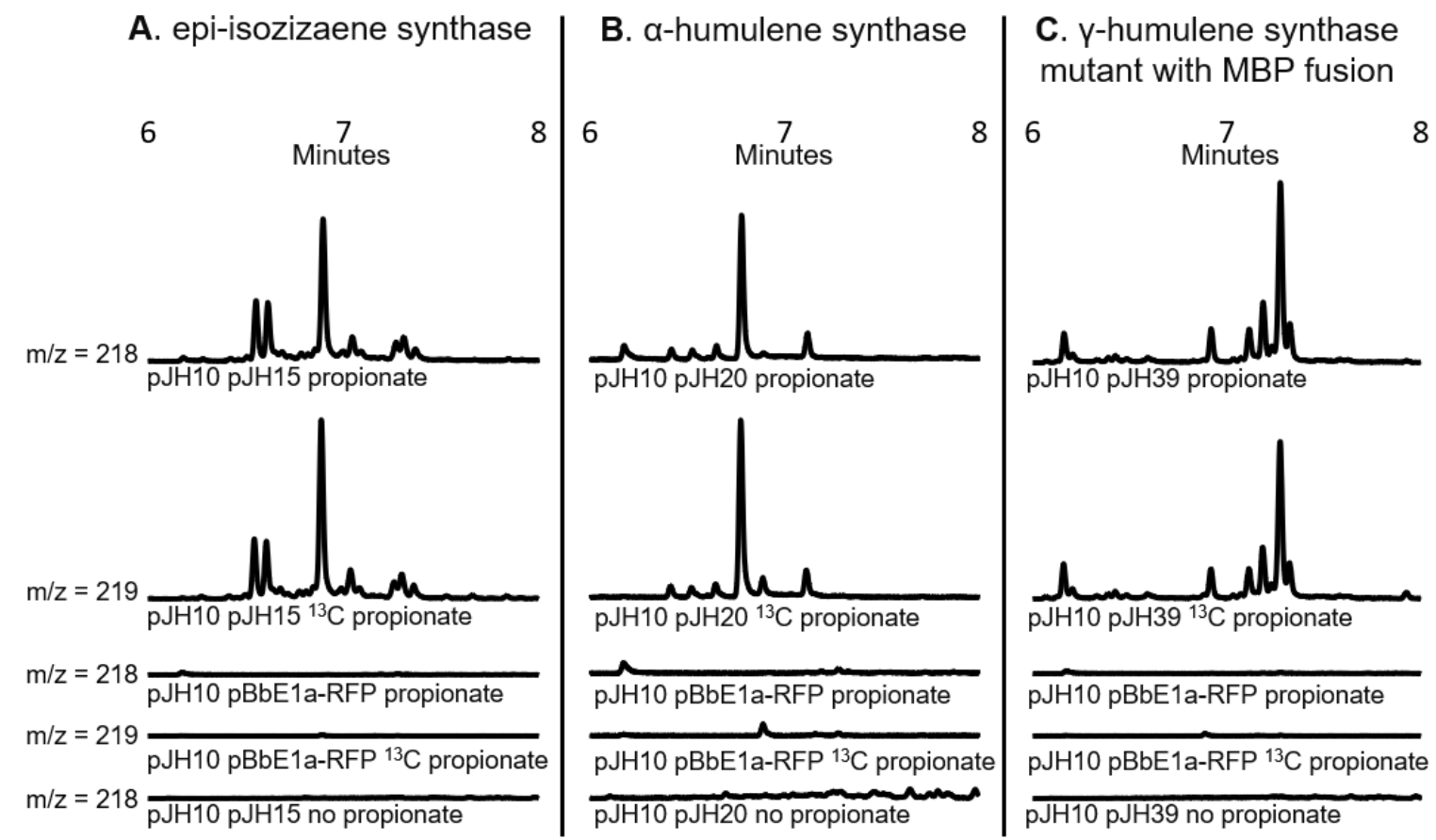

Fig. 2. GC-MS traces of nonane overlays of Bap1 E. coli cells expressing the lepidopteran mevalonate pathway and a terpene cyclase. Plasmid pJH10 contains the core enzymes of the pathway up to but not including the FPP synthases or a terpene cyclase. Plasmid pJH15, pJH20, and $\mathrm{pJH} 39$ all express the FPP synthases and a single terpene cyclase, either epi-isozizaene synthase (A), $\alpha$-humulene synthase (B), or a N-terminal maltose binding protein (MBP) fusion to $\gamma$-humulene synthase mutant S484A, Y566F (C). Plasmid pBbE1a-RFP expresses only RFP as a negative control. Fermentations were conducted with either no propionate, ${ }^{12} \mathrm{C}$ propionate or singly labeled ${ }^{13} \mathrm{C}$ propionate at $1 \mathrm{~g} / \mathrm{L}$. Traces show Selected Ion Monitoring (SIM) of $\mathrm{m} / \mathrm{z}=218$ for no propionate and propionate conditions, while $\mathrm{m} / \mathrm{z}=219$ is used for singly labeled ${ }^{13} \mathrm{C}$ propionate conditions.

To further investigate these novel compounds we scaled up our best producing strain (Bap1 pJH10 pJH15) to 500-mL culture volume with a $50 \mathrm{~mL}$ nonane overlay. After expressing per the usual protocol, we concentrated the nonane to approximately $100 \mu \mathrm{L}$ using a rotary evaporator under reduced pressure. We then analyzed the samples with high-resolution GCQTOF mass spectrometry. We confirmed the novel peaks have precursor and fragment ions consistent with $\mathrm{C}_{16} \mathrm{H}_{26}$ terpenes. The heaviest predicted ion has a $\mathrm{m} / \mathrm{z}=218.2029$ (Fig. 3A). The $203.1794 \mathrm{~m} / \mathrm{z}$ fragments correspond to sixteen carbon terpenes with loss of a methyl group. Similarly, the $189.1638 \mathrm{~m} / \mathrm{z}$ fragments corresponds to an ethyl group loss and $175.1481 \mathrm{~m} / \mathrm{z}$ to a propyl group loss (Fig. 3B).

The mass error of the novel terpenes parent ion on the GC-QTOF were [peak A $3.2 \mathrm{ppm}$ ], [peak B $1.37 \mathrm{ppm}$ ], and [peak C $-.92 \mathrm{ppm}$ ] with an average error of $1.83 \mathrm{ppm}$ over the three. We estimated the mass error across the observed fragmentation patterns against the theoretical 
fragmentations from $\mathrm{m} / \mathrm{z}=218.2029,203.1794,189.1638$ down to $\mathrm{m} / \mathrm{z} 91.054$ (loss of nine carbons). The average mass error was found to be [peak A $2.11 \mathrm{ppm}$ ], [peak B $1.28 \mathrm{ppm}$ ] and [peak C $1.05 \mathrm{ppm}$ ], with an average of $1.48 \mathrm{ppm}$ between the three. A $5 \mathrm{ppm}$ mass error is generally considered descent for confirming a molecular formula and 3 ppm excellent ${ }^{38}$. Given these data, we confirm the novel compounds have a molecular formula of $\mathrm{C}_{16} \mathrm{H}_{26}$.

A. Novel Terpenes Produced by Bap1 pJH10 pJH15 Detected by GC-QTOF TIC
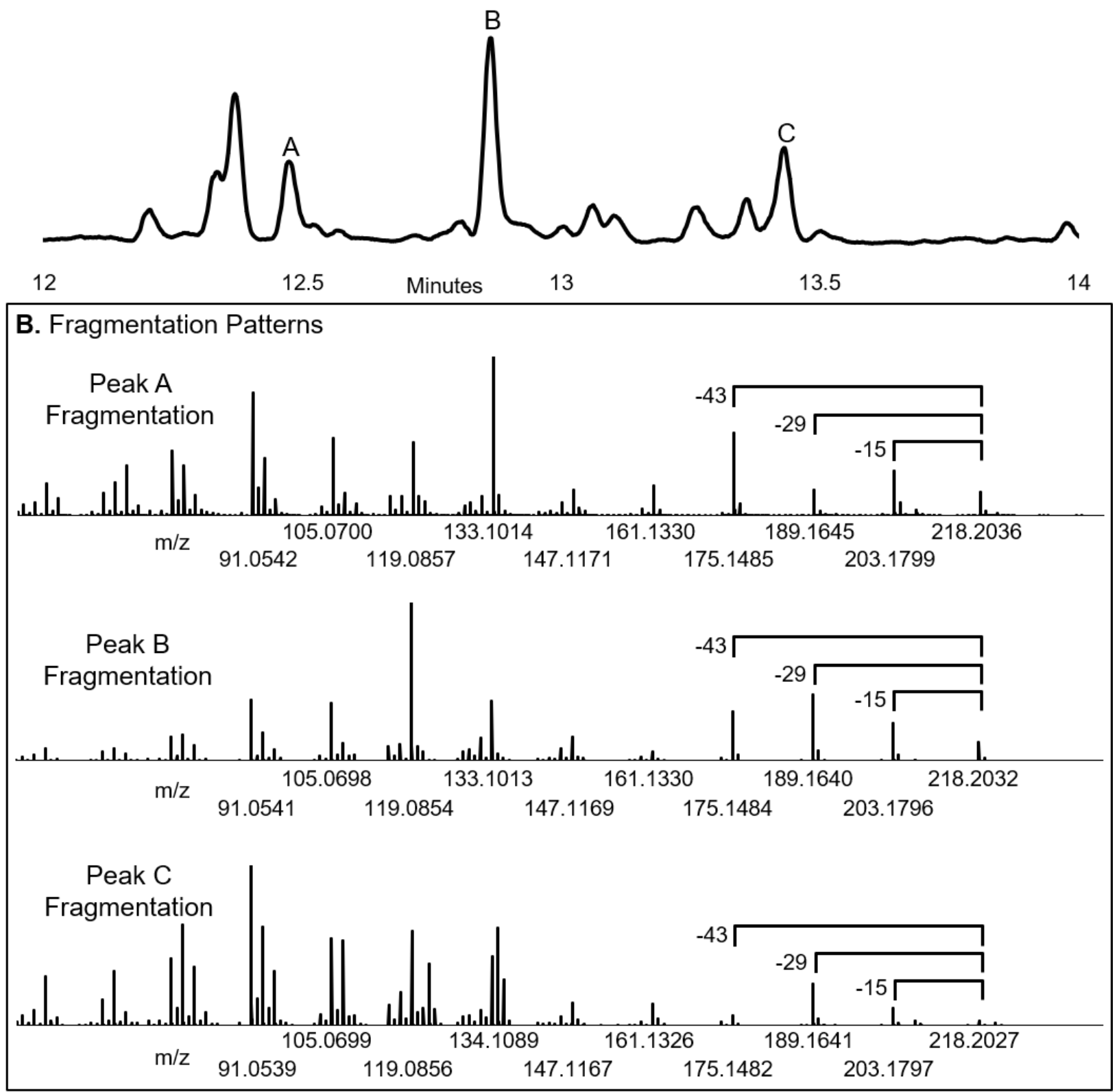

Fig. 3. GC-QTOF MS assays of novel terpenes produced by Bap1 pJH10 pJH15 cells. A. Total ion chromatogram of concentrated nonane overlay of Bap1 E. coli cells expressing the lepidopteran mevalonate pathway and an epi-isozizaene synthase from plasmids pJH10, pJH15. B. Fragmentation patterns of selected chromatographic peaks. Fragmentation patterns were averaged over the whole chromatographic peak. Ions consistent with $\mathrm{C}_{16} \mathrm{H}_{26}$ fragmentation have 
predicted $\mathrm{m} / \mathrm{z}$ of $218.2029,203.1794,189.1638,175.1481$ corresponding to the loss of an electron, a methyl group, an ethyl group and a propyl group respectively. Fragmentation patterns for the other chromatographic peaks can be found in the Dataset S1.

Estimating the novel compound titers is difficult because standards are not commercially available, nor have we successfully purified the novel compounds to homogeneity. A rough estimate can be made using the model sesquiterpene $\beta$-caryophyllene as a standard, but we stress the error in this crude estimate is hard to know a priori. Bearing in mind this caveat, we estimate that the combined $\mathrm{C}_{16} \mathrm{H}_{26}$ fraction for $\mathrm{pJH15}$, pJH20, pPJH39 per liter of cell culture to be 684 $\mathrm{mg}+/-.143 \mathrm{mg}(\mathrm{SD}), .081 \mathrm{mg}+/-.007 \mathrm{mg}$, and $.398 \mathrm{mg}+/-.042 \mathrm{mg}$, respectively.

We then tested if the lepidopteran mevalonate pathway uniquely accepts propionyl-CoA into higher terpenes, or if the phenomenon is more general. We used the plasmid pBbA5cMevT(CO)-T1-MBIS(CO, ispA) ${ }^{12}$, which expresses the Saccharomyces cerevisiae mevalonate pathway genes, and replaced the thiolase with PhaA from pJH10 to form pJH122. We cotransformed pJH122 with pJH15 into Bap1 and performed expressions as before. Concurrently we also assayed sesquiterpene $(\mathrm{m} / \mathrm{z}=204)$ vs homosesquiterpene $(\mathrm{m} / \mathrm{z}=218)$ production using the previous plasmid combinations to compare the percentage of novel terpenes versus the total terpene content, heeding the aforementioned caveat about difficulties of estimating concentrations without standards. Surprisingly, we found that Bap1 pJH122 pJH15 produced $0.16 \mathrm{mg} / \mathrm{L}+/-.12 \mathrm{mg} / \mathrm{L}$ (SD) novel homosesquiterpenes, about $16 \%$ as much as Bap1 pJH10 pJH15, which produced $1.05 \mathrm{mg} / \mathrm{L}+/-.219 \mathrm{mg} / \mathrm{L}$ in this experiment. We found that Bap1 pJH10 paired with pJH15, pJH20, and pJH39 made $3.31 \%+/-.88 \%, 5 \%+/-1.93 \%$ and $11.25 \%+/-$ 5.93\% homosesquiterpenes, respectively (Fig. 4), while Bap1 pJH122 pJH15 made .67\% +/$.30 \%$. 
Percent $\mathrm{C}_{16} \mathrm{H}_{26}$ Terpenes Compared to

Total Terpene Content

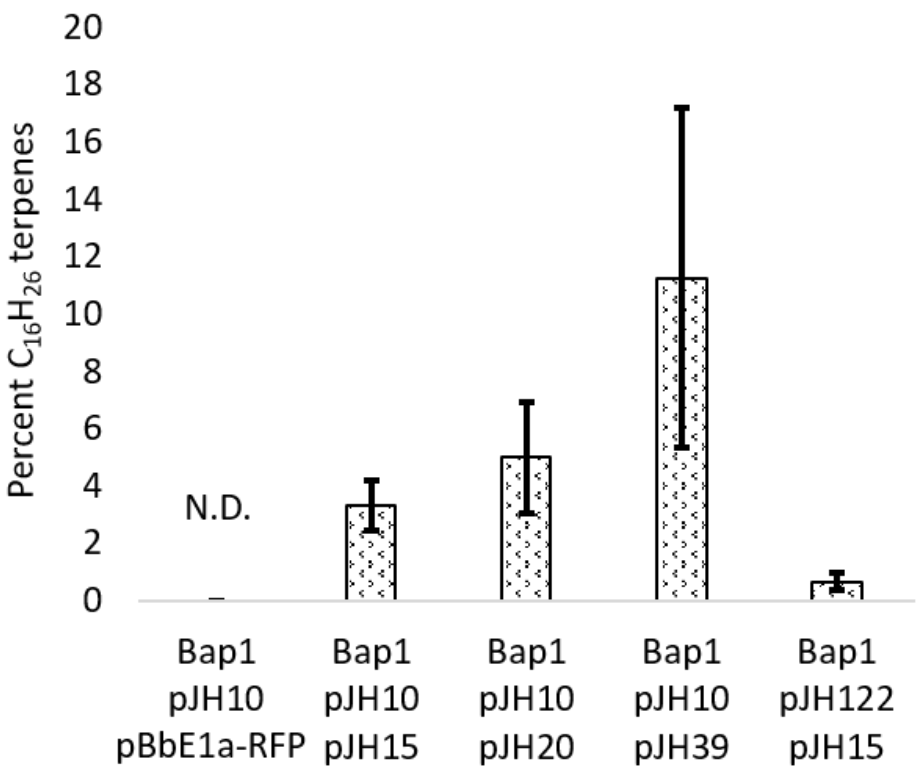

Fig. 4. Percentage of total terpenes that are $\mathrm{C}_{16} \mathrm{H}_{26}$ terpenes $\left(\mathrm{C}_{16} \mathrm{H}_{26} /\left(\mathrm{C}_{16} \mathrm{H}_{26}+\mathrm{C}_{15} \mathrm{H}_{24}\right)\right)$ produced by various strains. Error bars represent standard deviation of biological triplicates, except for Bap1 pJH122 pJH15, which represents nine biological replicates. N.D. indicates that neither sesquiterpenes nor homosesquiterpenes were detected.

Discussion. Although terpenoid compounds containing non multiple of five carbons are promising for pharmaceuticals, flavors, fragrances, commodity chemicals, and fuels, historically efficiently sampling this space has been difficult. In this paper we developed a homosesquiterpene diversity platform that can explore this novel chemical space. The most immediate application is sesquiterpene drug diversification. For instance, emerging resistance to the sesquiterpene antimalarial drug artemisinin will require new drug development to combat and underscores the importance of methods like ours which generate new analogues and eventually drug leads ${ }^{39}$.

Several methods confirmed the novel chemicals we produced here as

homosesquiterpenes. The chromatographic peaks incorporated ${ }^{13} \mathrm{C}$ propionate (parent ion $+1 \mathrm{~m} / \mathrm{z}$ shift compared to ${ }^{12} \mathrm{C}$ propionate), revealing the extra carbon originates from propionate as predicted. Unsurprisingly, propionate omission abolished the novel chromatographic peaks, as did replacing the terpene cyclase plasmid with an RFP expressing plasmid. Replacing epiisozizaene synthase with alternate terpene cyclases generated novel product profiles demonstrating this pathway can potentially generate large chemical diversity with predictable and minimal alterations.

We initially tried purifying the novel terpenes from the best producing strain (Bap1 pJH10 pJH15) for nuclear magnetic resonance (NMR) analysis but separating them from the 
nonane overlay and each other proved technically challenging. This is unsurprising as the novel terpene content compared to total terpene content was modest at $11.25 \%+/-5.93 \%$ (SD) in the best-case scenario for Bap1 pJH10 pJH39 and only 3.31\% +/- .88\% for Bap1 pJH10 pJH15, which produced our best titer. Therefore, we used GC-QTOF to validate the new molecules' chemical formulae, albeit without the atomic spatial configuration. We observed the parent ions (average mass error of $1.83 \mathrm{ppm}$ ) and the fragmentation ions (average mass error of 1.48) in great agreement with the predicted $\mathrm{m} / \mathrm{z} .5 \mathrm{ppm}$ mass error is generally considered good and 3 ppm is excellent for GC-QTOF. This confirmed the novel molecules exact composition as $\mathrm{C}_{16} \mathrm{H}_{26}$ and thus homosesquiterpenes. We found that the pathway can accept dissimilar terpene cyclases and still produce novel compounds, a beneficial property for terpene cyclase library screening. Epi-isozizaene synthase and $\alpha$-humulene synthase canonically produce very narrow product profiles $^{30,40,}$ while $\gamma$-humulene synthase mutant S484A, Y566F has a much broader product profile ${ }^{37}$. The three terpene cyclases also catalyze various degrees of FPP cyclization, with epi-isozizaene synthase producing a tricyclic compound, $\gamma$-humulene synthase mutant S484A, Y566F primarily making a variety of tricyclic compounds, and $\alpha$-humulene synthase primarily making a monocyclic compound. Despite these differences in fifteen carbon product profile, all made many sixteen carbon products. Given Juvenile Hormone natural diversity (Fig. 1D $)^{41}$, extending the strategy to seventeen and eighteen carbon terpenes should be possible, although we did not confirm their production here.

We expected only the lepidopteran mevalonate pathway to uniquely make homosesquiterpenes. However, we found the $S$. cerevisiae core mevalonate pathway enzymes, from HMGR to IDI, could incorporate propionyl-CoA when paired with an appropriate thiolase, the lepidopteran FPP synthases, and a terpene cyclase. However, the lepidoptera pathway counterpart made 6.6 times as much homosesqiterpenes as the $S$. cerevisiae pathway. The lepidopteran pathway also showed better specificity towards homosesqiterpenes than the $S$. cerevisiae pathway. A few known compounds from ant ${ }^{26}$ and other insects closely related to lepidoptera ${ }^{25}$ hint that mevalonate pathway incorporation of propionyl-CoA might not be strictly limited to lepidoptera. However, it is surprising that the mevalonate pathway from the much more divergent $S$. cerevisiae accepts propionyl-CoA. Perhaps more effort is justified in searching the canonical mevalonate pathway in nature for noncanonical terpenes.

Recently, a SAM methyltransferases strategy adding methyl groups to prenyl

pyrophosphates has enabled novel terpene production, including sesuiterpenes ${ }^{19,20,21,22}$. The SAM methyltransferase strategy is complimentary to the promiscuous mevalonate pathway because these two approaches incorporate methyl groups at different locations. The SAM methyltransferase strategy is ATP intensive as SAM regeneration requires hydrolyzing 3 high energy phosphate bonds ${ }^{42}$. The promiscuous mevalonate pathway does not require additional ATP compared to making traditional terpenes, but the propionyl-CoA need will require additional engineering to biosynthesize from popular carbon sources if the end application is making inexpensive commodity chemicals and fuels. 
To our knowledge this work is the first report using the lepidopteran mevalonate pathway to heterologously produce novel sixteen carbon terpenes, unlocking new space in the economically important terpenes compound class.

\section{Methods:}

Chemicals. Chemicals were ordered as follows: nonane and sodium propionate from Alfa Aesar; DpnI and T4 DNA ligase (5 Weiss U/ $\mu \mathrm{L}$ ) from Thermo Scientific; Molecular grade BSA, type IIS restriction enzyme BsaI and Gibson Assembly Master Mix from New England BioLabs; TB dry powder from EMD Millipore; IPTG from Fisher Scientific; Glucose from VWR Analytical; all other chemical reagents, including ${ }^{13} \mathrm{C}$ sodium propionate, from Sigma Aldrich. Qiagen QIAquick Gel Extraction Kits/PCR Purification kits, Zymo Research DNA Clean and Concentrator Kits and Millipore Nitrocellulose 0.025 $\mu \mathrm{M}$ VSWP dialysis membranes were used for DNA manipulations.

DNA synthesis and sources. DNA encoding bmHMGS, bmHMGR, bmMevK, bmMevPK, bmMevPPD, bmIPPI, and cfFPPS1 was codon optimized (Genescript) for E. coli, synthesized and cloned by Genescript into pET29b+ vector between the NdeI and XhoI cut sites harboring a TEV protease sequence before a C-terminal 6xHis tag. The amino acid sequences for "bm" constructs originate from Bombyx mori (silkworm), and those for "cf" from Choristoneura fumiferana (eastern spruce budworm). We sourced the thiolase PhaA from Acinetobacter strain RA3849 plasmid pMMB-pha ${ }^{31}$. Epi-Isozizaene synthase from Streptomyces coelicolor A3(2) was codon optimized using GeneDesign ${ }^{43}$ and synthesized by IDT as a gBlock. cfFPPS 2 native sequence was ordered through IDT as a gBlock $^{44}$.

PCR and Plasmid Assemblies. Primers for DNA amplification and construction were designed using DeviceEditor ${ }^{45}$ and/or $\mathrm{j}^{46}$ and are listed in Dataset S1. Touchdown PCR was used to amplify DNA fragments for plasmid construction of pET28cfFPPS2, pET28scEpiisozizaene, pET29bmHMGRt, pJH10, pJH15, pJH20, and pJH39. iProof was used as the polymerase using the manufacturer's recommended buffer conditions with the addition of 5\% dimethyl sulfoxide (DMSO). PCR reaction cycle was as follows: $98^{\circ} \mathrm{C}$ incubation for 30 seconds for one cycle. 10 cycles of $98^{\circ} \mathrm{C}$ for 10 seconds, annealing temperature (various, see Dataset S1) for 15 seconds, decreasing $.5^{\circ} \mathrm{C}$ each cycle, then an extension time of 22.5 seconds per kilobase at $72^{\circ} \mathrm{C}$. Then 25 cycles starting with a $98^{\circ} \mathrm{C}$ for 10 seconds, a constant annealing temperature $5^{\circ} \mathrm{C}$ lower than the initial annealing temperature for 15 seconds then an extension time of 22.5 seconds per $\mathrm{kb}$ at $72^{\circ} \mathrm{C}$. One final extension step of $10 \mathrm{~min}$ at $72^{\circ} \mathrm{C}$ was included at the end of the cycle before the samples were chilled to $4^{\circ} \mathrm{C}$.

PCR products were purified via a QIAquick PCR Purification kit, eluted with supplied EB buffer, DpnI digested at $37^{\circ} \mathrm{C}$ for 1 hour, followed by gel electrophoresis on a $.5 \%$ agarose gel using GelGreen nucleotide stain. Gel imaging was done with an Invitrogen Safe Imager. DNA bands of the correct size were excised and purified via a QIAquick Gel Extraction kit, then 
concentrated to $10 \mu \mathrm{L}$ using a Zymo Research DNA Clean and Concentrator kit. DNA concentration was measured using a NanoDrop at $260 \mathrm{~nm}$.

Gibson Assembly was used to construct plasmids pET28cfFPPS2, pET28scEpiisozizaene and, pET29bmHMGRt ${ }^{47}$ using Gibson Assembly Master Mix purchased from New England Biolabs. Equal molar amounts of inserts and backbone were added and incubated at $50^{\circ} \mathrm{C}$ for 1 hour. $1 \mu \mathrm{L}$ of reaction mixture was then electroporated using Electromax DH10B and plated on appropriate antibiotic. Compared to the full length sequence of bmHMGR, the first 390 amino acids of bmHMGRt were truncated, yielding MKKRPMV ... as the translational start of the protein based on structural intuition from the human HMGR1 crystal structure 2Q6B ${ }^{48}$.

pJH10 and pJH15 were constructed using Golden Gate Assembly ${ }^{49}$ using the BsaI enzyme. Assembly reaction condition consisted of .33 $\mu \mathrm{L}$ BsaI, $.33 \mu \mathrm{L}$ T4 DNA ligase, $0.05 \mu \mathrm{L}$ BSA, $.5 \mu \mathrm{L} \mathrm{10x} \mathrm{T4} \mathrm{ligase} \mathrm{buffer,} 33$ ng of backbone, and the other assembly pieces were added in equimolar concentration to the backbone. Water was then added to $5 \mu \mathrm{L}$ total volume. The assembly mixture used a thermocycling protocol of $45^{\circ} \mathrm{C}$ for 2 minutes, $16^{\circ} \mathrm{C}$ for 5 minutes, cycled 25 times, followed by $50^{\circ} \mathrm{C}$ for 5 minutes, $80^{\circ} \mathrm{C}$ for 5 minutes, and $4^{\circ} \mathrm{C}$ until samples were recovered from the PCR blocks.

pJH15 was used as the template to produce pJH20 and pJH39 via Golden Gate Assembly. The epi-isozizaene synthase from pJH15 was replaced with the terpene cyclases $\alpha$-humulene synthase from Zingiber zerumbet (bitter ginger) from plasmid pTrcHumulene (expressing ZSS1) for $\mathrm{pJH} 20^{36}$. A N-terminal Maltose Binding Protein (MBP) fusion from $\mathrm{pET} 28 \mathrm{a}-\mathrm{MBP}$ (previously unpublished plasmid made by Dr. Andrew Hagen, Ph.D.) was cloned in front of a $\gamma$ humulene synthase mutant S484A, Y566F from Abies grandis (grand fir) from plasmid pTrcHUM_Y566F_484A (AYG from table 1) ${ }^{37}$ for pJH39. Plasmids will be available upon request from authors or Addgene.

Plasmid confirmation via MiSeq sequencing. Plasmid deep sequencing was performed as in previous work ${ }^{50}$.

E. coli KCM competent cell prep. Competent cells were prepared starting from a single colony or glycerol stock on day one into an LB overnight. On day two the overnight was diluted 1:100 into $50 \mathrm{~mL} \mathrm{LB}$ and grown at $37^{\circ} \mathrm{C}$ shaking at $200 \mathrm{RPM}$ or higher to an OD 600 of 0.35 as measured with a Beckman Coulter DU800 spectrophotometer. Cells were then chilled on ice for 20 minutes before centrifugation at $8000 \mathrm{rcf}$ for 8 minutes. The supernatant was decanted, and cells resuspended in $5 \mathrm{~mL}$ of ice-cold sterile-filtered TSS (LB base with 10\% PEG 3350 (w/v), $5 \%$ DMSO (v/v), $20 \mathrm{mM} \mathrm{MgCl}_{2}$ ). $100 \mu \mathrm{L}$ of TSS and cell mixture were then aliquoted and used immediately or flash frozen using liquid nitrogen and then stored at $-80^{\circ} \mathrm{C}$ till use.

$1 \mu \mathrm{L}$ of each desired plasmid was added to the TSS cell mixture and mixed with $100 \mu \mathrm{L}$ of sterile $2 \mathrm{x} \mathrm{KCM}\left(0.2 \mathrm{M} \mathrm{KCl}, 0.06 \mathrm{M} \mathrm{CaCl}_{2}, 0.1 \mathrm{M} \mathrm{MgCl}_{2}\right)$ and incubated on ice for 20 minutes. Cells were then heat shocked at $42^{\circ} \mathrm{C}$ for 90 seconds before being recovered with 200 $\mu \mathrm{L} \mathrm{TB}$ at $37^{\circ} \mathrm{C}$ for one hour. Cells were then plated onto LB agar plates with appropriate 
antibiotics (carbenicillin at a final concentration of $100 \mu \mathrm{g} / \mathrm{mL}$, and chloramphenicol at a final concentration of $25 \mu \mathrm{g} / \mathrm{mL})$.

E. coli pathway expressions. Freshly transformed colonies were picked into $10 \mathrm{~mL}$ Terrific Broth medium (yeast extract $24 \mathrm{~g} / \mathrm{L}$, tryptone $20 \mathrm{~g} / \mathrm{L}$, glycerol $4 \mathrm{~mL} / \mathrm{L}, 17 \mathrm{mM} \mathrm{KH}_{2} \mathrm{PO}_{4}, 72 \mathrm{mM}$ $\mathrm{K}_{2} \mathrm{HPO}_{4}$ ) with $0.4 \%$ glucose and incubated shaking overnight in glass test tubes. Cells from overnights were diluted 1:100 into fresh TB with $0.4 \%$ glucose and $1 \mathrm{~g} / \mathrm{L}$ sodium propionate (unlabeled or $1-{ }^{13} \mathrm{C}$ labeled). Cells were grown at $200 \mathrm{RPM}$ and $37^{\circ} \mathrm{C}$ until OD600 of 0.8. IPTG was then added to a final concentration of $1 \mathrm{mM}$ and a nonane overlay was added. Temperature was reduced to $18^{\circ} \mathrm{C}$ and the cells were incubated for 36 hours in small scale experiments, and 30 hours for the large scale experiment before harvesting. For small scale expressions, the culture volume was $10 \mathrm{~mL}$ in glass test tubes with $1 \mathrm{~mL}$ nonane overlay, and large scale expressions were done at $500 \mathrm{~mL}$ in baffled flasks with $50 \mathrm{~mL}$ nonane overlay.

Small scale samples. $1.4 \mathrm{~mL}$ of the nonane/culture emulsions were harvested and centrifuged at $18000 \mathrm{rcf}$ for $1 \mathrm{~min}$ to separate the organic layer. $100 \mu \mathrm{L}$ of the organic layer was collected and 1 $\mu \mathrm{L}$ analyzed directly by GC-MS. Samples were stored at $-20^{\circ} \mathrm{C}$ for short term (one to two days), or at $-80^{\circ} \mathrm{C}$ if longer term storage was necessary in amber glass vials.

Large scale enrichment. The cell culture was added to a separation funnel and allowed to sit until the overly and a foamy layer separated from the aqueous layer. The aqueous layer was removed, leaving a foamy layer and an organic layer. These layers were washed with approximately $200 \mathrm{~mL} \mathrm{NaCl}$ saturated water. After the layers separated the aqueous layer was removed. This wash step was repeated one time. The foamy layer and organic layer were put into $50 \mathrm{~mL}$ conical tubes and spun at 10,000 rcf for $3 \mathrm{~min}$. After centrifugation the foamy layer was greatly reduced and instead a white "goopy" layer appears between the organic layer and aqueous layer. The organic layer was then transferred to a round bottomed flask and a rotary evaporator was used under reduced pressure concentrate the sample to approximately $100 \mu \mathrm{L}$. This liquid was sent through a glass wool mesh to remove any particulate. Samples were stored at $-80^{\circ} \mathrm{C}$ or on dry ice under a nitrogen atmosphere until analysis.

GC-MS analysis method. Samples were run on an Agilent $6890 \mathrm{GC}$ and Agilent 5973 MS using a 30-meter DB-5ms column with a helium flow of $1 \mathrm{~mL} / \mathrm{min}$. The $\mathrm{GC}$ was run in splitless mode with a constant inlet temp and transfer line temp of $280^{\circ} \mathrm{C}$. The oven starting temperature was $100^{\circ} \mathrm{C}$ held for 1 minute. The temperature was increased at $20^{\circ} \mathrm{C}$ per minute until $160^{\circ} \mathrm{C}$. Then the temperature was increased at $10^{\circ} \mathrm{C}$ per minute until $225^{\circ} \mathrm{C}$. Then the temperature was increased at $30^{\circ} \mathrm{C}$ per minute until $300^{\circ} \mathrm{C}$ where it was held for $2 \mathrm{~min}$. Single ion monitoring was carried out at 218 or $219 \mathrm{~m} / \mathrm{z}$ as appropriate to detect ${ }^{12} \mathrm{C}$ or ${ }^{13} \mathrm{C}$ labeled products. A relative gain factor of 2 was used. Data was analyzed via Chemstation Enhanced Data Analysis program. For peak integration the RTE integrator was used. 
GC-QTOF analysis method. Accurate mass GC-QTOF data was collected on an Agilent 7890A GC coupled to an Agilent 7200 QTOF mass spectrometer (Agilent Technologies, CA, USA). For chromatographic separation, a Restek RTx-5Sil MS column (30 m x 0.25 mm, $0.25 \mu \mathrm{m}$; Restek Corporation, PA, USA) was used with Helium (99.999\%; Airgas, PA, USA) flow maintained at $1 \mathrm{~mL} / \mathrm{min}$. Oven temperature started at $60^{\circ} \mathrm{C}$ for $1 \mathrm{~min}$, increased at a rate of $10^{\circ} \mathrm{C} / \mathrm{min}$ to $325^{\circ} \mathrm{C}$ and held constant for $9.5 \mathrm{~min}$, with a transfer line temperature of $270{ }^{\circ} \mathrm{C}$. Spectra were collected over a range of $50-800 \mathrm{~m} / \mathrm{z}$ at a rate of 5 spectra per second for electron ionization (EI). The source was maintained at $230^{\circ} \mathrm{C}$, with $70 \mathrm{eV}$ electron energy. The transfer line temperature was $270^{\circ} \mathrm{C}$. Data was analyzed using MassHunter Qualitative Analysis software (Agilent Technologies, CA, USA).

Proteomics. Cells were grown as in the expressions above. 20 OD mLs of cells were collected and proteomics carried out essentially as described in previous work ${ }^{51}$. Briefly, lysis of E. coli cell pellets combined with protein precipitation was achieved by the addition of $80 \mu \mathrm{L}$ of methanol, $20 \mu \mathrm{L}$ of chloroform, and $60 \mu \mathrm{L}$ of water, with vortexing. The samples were centrifuged at 20,817 x g for 1 minute for phase separation. The hydrophilic methanol and water (top) layer was removed, then $100 \mu \mathrm{L}$ of methanol was added and the sample was vortexed briefly followed by centrifugation for 1 minute to isolate the protein pellet. The protein pellet was resuspended in $100 \mathrm{mM}$ ammonium bicarbonate with $20 \%$ methanol and quantified by the Lowry method. A total of $100 \mu \mathrm{g}$ of protein was reduced by adding tris 2-(carboxyethyl) phosphine (TCEP) to a final concentration of $5 \mathrm{mM}$, alkylated by adding iodoacetamide at a final concentration of $10 \mathrm{mM}$, and digested overnight at $37^{\circ} \mathrm{C}$ with trypsin at a ratio of 1:50 (w/w) trypsin:total protein.

Peptides were analyzed using an Agilent 1290 liquid chromatography system coupled to an Agilent 6460QQQ mass spectrometer (Agilent Technologies, Santa Clara, CA) operating in MRM mode. Peptide samples $(10 \mu \mathrm{g})$ were separated on an Ascentis Express Peptide ES-C18 column $\left(2.7 \mu \mathrm{m}\right.$ particle size, $160 \AA$ pore size, $50 \mathrm{~mm}$ length x $2.1 \mathrm{~mm}$ i.d., $60{ }^{\circ} \mathrm{C}$; SigmaAldrich, St. Louis, MO) by using a chromatographic gradient ( $400 \mu \mathrm{L} / \mathrm{min}$ flow rate) with an initial condition of $95 \%$ Buffer A (99.9\% water, $0.1 \%$ formic acid) and 5\% Buffer B (99.9\% acetonitrile, $0.1 \%$ formic acid) then increasing linearly to $65 \%$ Buffer A/35\% Buffer B over 5.5 minutes. Buffer B was then increased to $80 \%$ over 0.3 minutes and held at $80 \%$ for two minutes followed by ramping back down to $5 \%$ Buffer B over 0.5 minutes where it was held for 1.5 minutes to re-equilibrate the column for the next sample. The data were acquired using Agilent MassHunter, version B.08.02, processed using Skyline version 4.1, and peak quantification was refined with mProphet in Skyline. All data and skyline files are available via the Panorama Public repository at this link: https://panoramaweb.org/Heterologous-Lepidopteran-MevalonatePathway.url. Data are also available via ProteomeXchange with identifier PXD012697. 
Method for novel terpene titer estimation. Estimating titers of the homo-terpene compounds is difficult because we cannot buy standards, nor have we been able to successfully purify them to homogeneity. To make rough estimates we used $\beta$-caryophyllene as a standard. However, we stress the error in this estimate is hard to know a priori as we must assume the novel terpenes ionize like the parent ion of $\beta$-caryophyllene. $1 \mu \mathrm{L}$ of $\beta$-caryophyllene was diluted into $99 \mu \mathrm{L}$ of ethyl acetate (or in another instance $9 \mathrm{uL}$ ethyl acetate). This mixture was then diluted $1.5 \mu \mathrm{L}$ into $1500 \mu \mathrm{L}$ ethyl acetate yielding a vial of $0.01 \mu \mathrm{L} \beta$-caryophyllene per mL of ethyl acetate (or $.1 \mu \mathrm{L} \beta$-caryophyllene per $\mathrm{mL}$ of ethyl acetate in another instance). $750 \mu \mathrm{L}$ of this mixture was serially diluted to generate the standard curve. The standard curve was run from the smallest concentration vial to the vial with highest concentration using GC-MS in single ion monitoring mode detecting $\mathrm{m} / \mathrm{z}=204$. $\beta$-caryophyllene density was taken to be $0.9052 \mathrm{~g} / \mathrm{cm}^{3}$ at room temperature (collected from https://en.wikipedia.org/wiki/Caryophyllene).

To estimate the purity of $\beta$-caryophyllene, a sample of $0.01 \mu \mathrm{L} \beta$-caryophyllene per $\mathrm{mL}$ of ethyl acetate (or .1 $\mu \mathrm{L} \beta$-caryophyllene per $\mathrm{mL}$ of ethyl acetate in another instance) was analyzed using scan mode on GC-MS. The sample contained primarily $\beta$-caryophyllene but also some amount of two oxidized $\beta$-caryophyllene compounds. By taking the ratio of the peak areas we concluded that standard was $94.4 \% \beta$-caryophyllene, and the 5.6\% other compounds and (and in another instance using new $\beta$-caryophyllene, $97.4 \%$ and $2.62 \%$ other compounds). The excel function LINEST was used to generate an equation to convert peak area to $\mathrm{mg} / \mathrm{L}$, we then corrected for the purity of our $\beta$-caryophyllene standard.

To estimate the homosesquiterpene peak areas we averaged the ${ }^{12} \mathrm{C}$ propionate and ${ }^{13} \mathrm{C}$ propionate fermentation runs, each in biological triplicate thus representing six observations from Dataset_S1.

Method for novel homosesquiterpene to total terpene fraction estimation. Peak areas for homosesuiterpene were gathered at $\mathrm{m} / \mathrm{z}=218$, and sesquiterpenes at $\mathrm{m} / \mathrm{z}=204$. These areas were then converted into the percent $\mathrm{C}_{16} \mathrm{H}_{26}$ terpenes compared to total terpenes $\left(\mathrm{C}_{16} \mathrm{H}_{26}\right.$ PeakArea / $\left(\mathrm{C}_{16} \mathrm{H}_{26}\right.$ PeakArea ${ }_{+} \mathrm{C}_{15} \mathrm{H}_{24}$ PeakArea $)$ within a biological replicate. Biological triplicate was used except for pJH122, which used nine replicates.

Author contributions. C.B.E. helped conceive and planned the project including, plasmid design, construction, experimental design, sample preparation, GC-MS assays as well as writing the primary draft of the paper. T.D.R. helped with GC-MS method development and homoterpene enrichment protocol as well as providing chemistry insights. C.B. provided GCQTOF method development and analysis. J.G. performed the proteomics assays. J.C. aided in deep sequencing of plasmids. E.E.K.B. provided data analysis and data representation help. C.J.P. oversaw the proteomics effort. N.J.H. contributed to manuscript revisions. O.F. oversaw GC-QTOF analysis. J.D.K. helped conceive and guide the project, as well as helping with the manuscript writing. 
Acknowledgements. C.B.E. would like to thank Marlin Eiben for thoughtful conversation on statistics, as well as Nurgul Kaplan and Garima Goyal for plasmid construction related to, but ultimately not included, in this manuscript.

Funding Sources. C.B.E. was supported by National Science Foundation (NSF) Graduate Research Fellowship Program (DGE-1106400) and National Institute of Health training grant T32 GM008295. NSF grant 0540879 supported the materials for this work. This work was part of the DOE Joint BioEnergy Institute (https://www.jbei.org) supported by the U. S. Department of Energy, Office of Science, Office of Biological and Environmental Research, and was part of the Agile BioFoundry (https://agilebiofoundry.org) supported by the U. S. Department of Energy, Energy Efficiency and Renewable Energy, Bioenergy Technologies Office, through contract DE-AC02-05CH11231 between Lawrence Berkeley National Laboratory and the U. S. Department of Energy. The views and opinions of the authors expressed herein do not necessarily state or reflect those of the United States Government or any agency thereof. Neither the United States Government nor any agency thereof, nor any of their employees, makes any warranty, expressed or implied, or assumes any legal liability or responsibility for the accuracy, completeness, or usefulness of any information, apparatus, product, or process disclosed, or represents that its use would not infringe privately owned rights. The United States Government retains and the publisher, by accepting the article for publication, acknowledges that the United States Government retains a non-exclusive, paid-up, irrevocable, world-wide license to publish or reproduce the published form of this manuscript, or allow others to do so, for United States Government purposes. The Department of Energy will provide public access to these results of federally sponsored research in accordance with the DOE Public Access Plan (http://energy.gov/downloads/doe-public-access-plan).

Data availability. Genbank files of all plasmids are available at https://publicregistry.jbei.org/folders/421. Plasmids will be available upon request from authors or Addgene. Dataset $\mathrm{S} 1$ is also available from the Experiment Data Depot ${ }^{52} \mathrm{https}: / /$ publicedd.jbei.org/accounts/login/?next=/s/exploring-non-canonical-terpene-space-with-aheter/overview/ The proteomics data has been uploaded to ProteomeXchange ID: $\underline{\text { PXD012697 }}$

Supplementary Information. The Dataset S1 contains GC-MS, GC-QTOF traces and fragmentation patterns, terpene quantification data, and plasmid construction details including primer sequences and PCR condition.

Competing Interest. The pathway has been patented by J.D.K. and C.B.E. J.D.K. has a financial interest in Amyris, Lygos, Demetrix, Maple Bio, and Napigen.

\section{Bibliography.}


(1) Ajikumar, P. K., Tyo, K., Carlsen, S., Mucha, O., Phon, T. H., and Stephanopoulos, G. (2008) Terpenoids: opportunities for biosynthesis of natural product drugs using engineered microorganisms. Mol. Pharm. 5, 167-190.

(2) Horton, J. D. (2002) Sterol regulatory element-binding proteins: transcriptional activators of lipid synthesis. Biochem. Soc. Trans. 30, 1091-1095.

(3) Unden, G., and Bongaerts, J. (1997) Alternative respiratory pathways of Escherichia coli: energetics and transcriptional regulation in response to electron acceptors. Biochim. Biophys. Acta 1320, 217-234.

(4) Zhang, F. L., and Casey, P. J. (1996) Protein prenylation: molecular mechanisms and functional consequences. Annu. Rev. Biochem. 65, 241-269.

(5) Prossnitz, E. R., and Maggiolini, M. (2009) Mechanisms of estrogen signaling and gene expression via GPR30. Mol. Cell. Endocrinol. 308, 32-38.

(6) Gershenzon, J., and Dudareva, N. (2007) The function of terpene natural products in the natural world. Nat. Chem. Biol. 3, 408-414.

(7) Kingston, D. G. I. (2007) The shape of things to come: structural and synthetic studies of taxol and related compounds. Phytochemistry 68, 1844-1854.

(8) Denby, C. M., Li, R. A., Vu, V. T., Costello, Z., Lin, W., Chan, L. J. G., Williams, J., Donaldson, B., Bamforth, C. W., Petzold, C. J., Scheller, H. V., Martin, H. G., and Keasling, J. D. (2018) Industrial brewing yeast engineered for the production of primary flavor determinants in hopped beer. Nat. Commun. 9, 965.

(9) Henke, N. A., Wichmann, J., Baier, T., Frohwitter, J., Lauersen, K. J., Risse, J. M., PetersWendisch, P., Kruse, O., and Wendisch, V. F. (2018) Patchoulol Production with Metabolically Engineered Corynebacterium glutamicum. Genes (Basel) 9.

(10) Lindberg, P., Park, S., and Melis, A. (2010) Engineering a platform for photosynthetic isoprene production in cyanobacteria, using Synechocystis as the model organism. Metab. Eng. $12,70-79$.

(11) Alonso-Gutierrez, J., Chan, R., Batth, T. S., Adams, P. D., Keasling, J. D., Petzold, C. J., and Lee, T. S. (2013) Metabolic engineering of Escherichia coli for limonene and perillyl alcohol production. Metab. Eng. 19, 33-41.

(12) Peralta-Yahya, P. P., Ouellet, M., Chan, R., Mukhopadhyay, A., Keasling, J. D., and Lee, T. S. (2011) Identification and microbial production of a terpene-based advanced biofuel. Nat. Commun. 2, 483.

(13) George, K. W., Thompson, M. G., Kang, A., Baidoo, E., Wang, G., Chan, L. J. G., Adams, P. D., Petzold, C. J., Keasling, J. D., and Lee, T. S. (2015) Metabolic engineering for the highyield production of isoprenoid-based $\mathrm{C}_{5}$ alcohols in E. coli. Sci. Rep. 5, 11128.

(14) Chou, H. H., and Keasling, J. D. (2012) Synthetic pathway for production of five-carbon alcohols from isopentenyl diphosphate. Appl. Environ. Microbiol. 78, 7849-7855.

(15) Jiang, J., He, X., and Cane, D. E. (2007) Biosynthesis of the earthy odorant geosmin by a bifunctional Streptomyces coelicolor enzyme. Nat. Chem. Biol. 3, 711-715. 
(16) Tsurumaru, Y., Sasaki, K., Miyawaki, T., Uto, Y., Momma, T., Umemoto, N., Momose, M., and Yazaki, K. (2012) HIPT-1, a membrane-bound prenyltransferase responsible for the biosynthesis of bitter acids in hops. Biochem. Biophys. Res. Commun. 417, 393-398.

(17) Wang, C.-M., and Cane, D. E. (2008) Biochemistry and molecular genetics of the biosynthesis of the earthy odorant methylisoborneol in Streptomyces coelicolor. J. Am. Chem. Soc. 130, 8908-8909.

(18) Ozaki, T., Shinde, S. S., Gao, L., Okuizumi, R., Liu, C., Ogasawara, Y., Lei, X., Dairi, T., Minami, A., and Oikawa, H. (2018) Enzymatic formation of a skipped methyl-substituted octaprenyl side chain of longestin (KS-505a): Involvement of homo-IPP as a common extender unit. Angew. Chem. Int. Ed. Engl. 57, 6629-6632.

(19) Drummond, L., Kschowak, M. J., Breitenbach, J., Wolff, H., Shi, Y.-M., Schrader, J., Bode, H. B., Sandmann, G., and Buchhaupt, M. (2019) Expanding the isoprenoid building block repertoire with an IPP methyltransferase from Streptomyces monomycini. ACS Synth. Biol. (20) Ignea, C., Pontini, M., Motawia, M. S., Maffei, M. E., Makris, A. M., and Kampranis, S. C. (2018) Synthesis of 11-carbon terpenoids in yeast using protein and metabolic engineering. Nat. Chem. Biol. 14, 1090-1098.

(21) Kschowak, M. J., Wortmann, H., Dickschat, J. S., Schrader, J., and Buchhaupt, M. (2018) Heterologous expression of 2-methylisoborneol / 2 methylenebornane biosynthesis genes in Escherichia coli yields novel C11-terpenes. PLoS One 13, e0196082. (22) von Reuss, S. H., Domik, D., Lemfack, M. C., Magnus, N., Kai, M., Weise, T., and Piechulla, B. (2018) Sodorifen biosynthesis in the rhizobacterium Serratia plymuthica involves methylation and cyclization of MEP-derived farnesyl pyrophosphate by a SAM-dependent Cmethyltransferase. J. Am. Chem. Soc. 140, 11855-11862.

(23) Schooley, D. A., Judy, K. J., Bergot, B. J., Hall, M. S., and Siddall, J. B. (1973)

Biosynthesis of the Juvenile Hormones of Manduca sexta: Labeling Pattern from Mevalonate, Propionate, and Acetate. Proc. Natl. Acad. Sci. USA 70, 2921-2925.

(24) Cascón, O., Touchet, S., Miller, D. J., Gonzalez, V., Faraldos, J. A., and Allemann, R. K. (2012) Chemoenzymatic preparation of germacrene analogues. Chem. Commun. 48, 9702-9704. (25) Hamilton, J. G. C., Hooper, A. M., Pickett, J. A., Mori, K., and Sano, S. (1999) 3-Methyl- $\alpha-$ himachalene is confirmed, and the relative stereochemistry defined, by synthesis as the sex pheromone of the sandfly Lutzomyia longipalpis from Jacobina, Brazil. Chem. Commun. 355356.

(26) Attygalle, A. B., and Morgan, E. D. (1982) Structures of homofarnesene and bishomofarnesene isomers from Myrmica ants. J. Chem. Soc., Perkin Trans. 1949. (27) Kinjoh, T., Kaneko, Y., Itoyama, K., Mita, K., Hiruma, K., and Shinoda, T. (2007) Control of juvenile hormone biosynthesis in Bombyx mori: cloning of the enzymes in the mevalonate pathway and assessment of their developmental expression in the corpora allata. Insect Biochem. Mol. Biol. 37, 808-818. 
(28) Sen, S. E., Cusson, M., Trobaugh, C., Béliveau, C., Richard, T., Graham, W., Mimms, A., and Roberts, G. (2007) Purification, properties and heteromeric association of type-1 and type-2 lepidopteran farnesyl diphosphate synthases. Insect Biochem. Mol. Biol. 37, 819-828.

(29) Sen, S. E., Tomasello, A., Grasso, M., Denton, R., Macor, J., Béliveau, C., Cusson, M., and Crowell, D. N. (2012) Cloning, expression and characterization of lepidopteran isopentenyl diphosphate isomerase. Insect Biochem. Mol. Biol. 42, 739-750.

(30) Aaron, J. A., Lin, X., Cane, D. E., and Christianson, D. W. (2010) Structure of epiisozizaene synthase from Streptomyces coelicolor A3(2), a platform for new terpenoid cyclization templates. Biochemistry 49, 1787-1797.

(31) Aldor, I. S., Kim, S.-W., Prather, K. L. J., and Keasling, J. D. (2002) Metabolic engineering of a novel propionate-independent pathway for the production of poly(3-hydroxybutyrate-co-3hydroxyvalerate) in recombinant Salmonella enterica serovar typhimurium. Appl. Environ. Microbiol. 68, 3848-3854.

(32) Polakowski, T., Stahl, U., and Lang, C. (1998) Overexpression of a cytosolic

hydroxymethylglutaryl-CoA reductase leads to squalene accumulation in yeast. Appl. Microbiol. Biotechnol. 49, 66-71.

(33) Ham, T. S., Dmytriv, Z., Plahar, H., Chen, J., Hillson, N. J., and Keasling, J. D. (2012)

Design, implementation and practice of JBEI-ICE: an open source biological part registry platform and tools. Nucleic Acids Res. 40, e141.

(34) Pfeifer, B. A., Admiraal, S. J., Gramajo, H., Cane, D. E., and Khosla, C. (2001)

Biosynthesis of complex polyketides in a metabolically engineered strain of E. coli. Science 291, 1790-1792.

(35) Lee, T. S., Krupa, R. A., Zhang, F., Hajimorad, M., Holtz, W. J., Prasad, N., Lee, S. K., and Keasling, J. D. (2011) BglBrick vectors and datasheets: A synthetic biology platform for gene expression. J. Biol. Eng. 5, 12.

(36) Xie, X., Kirby, J., and Keasling, J. D. (2012) Functional characterization of four sesquiterpene synthases from Ricinus communis (castor bean). Phytochemistry 78, 20-28.

(37) Yoshikuni, Y., Ferrin, T. E., and Keasling, J. D. (2006) Designed divergent evolution of enzyme function. Nature 440, 1078-1082.

(38) Draper, J., Enot, D. P., Parker, D., Beckmann, M., Snowdon, S., Lin, W., and Zubair, H. (2009) Metabolite signal identification in accurate mass metabolomics data with MZedDB, an interactive $\mathrm{m} / \mathrm{z}$ annotation tool utilising predicted ionisation behaviour "rules". BMC Bioinformatics 10, 227.

(39) Haldar, K., Bhattacharjee, S., and Safeukui, I. (2018) Drug resistance in Plasmodium. Nat. Rev. Microbiol. 16, 156-170.

(40) Yu, F., Okamto, S., Nakasone, K., Adachi, K., Matsuda, S., Harada, H., Misawa, N., and Utsumi, R. (2008) Molecular cloning and functional characterization of alpha-humulene synthase, a possible key enzyme of zerumbone biosynthesis in shampoo ginger (Zingiber zerumbet Smith). Planta 227, 1291-1299. 
(41) Kamita, S. G., Samra, A. I., Liu, J.-Y., Cornel, A. J., and Hammock, B. D. (2011) Juvenile hormone $(\mathrm{JH})$ esterase of the mosquito Culex quinquefasciatus is not a target of the $\mathrm{JH}$ analog insecticide methoprene. PLoS One 6, e28392.

(42) Markham, G. D., Hafner, E. W., Tabor, C. W., and Tabor, H. (1980) S-Adenosylmethionine synthetase from Escherichia coli. J. Biol. Chem. 255, 9082-9092.

(43) Richardson, S. M., Nunley, P. W., Yarrington, R. M., Boeke, J. D., and Bader, J. S. (2010) GeneDesign 3.0 is an updated synthetic biology toolkit. Nucleic Acids Res. 38, 2603-2606.

(44) Cusson, M., Béliveau, C., Sen, S. E., Vandermoten, S., Rutledge, R. G., Stewart, D., Francis, F., Haubruge, E., Rehse, P., Huggins, D. J., Dowling, A. P. G., and Grant, G. H. (2006) Characterization and tissue-specific expression of two lepidopteran farnesyl diphosphate synthase homologs: implications for the biosynthesis of ethyl-substituted juvenile hormones. Proteins 65, 742-758.

(45) Chen, J., Densmore, D., Ham, T. S., Keasling, J. D., and Hillson, N. J. (2012) DeviceEditor visual biological CAD canvas. J. Biol. Eng. 6, 1.

(46) Hillson, N. J., Rosengarten, R. D., and Keasling, J. D. (2012) j5 DNA assembly design automation software. ACS Synth. Biol. 1, 14-21.

(47) Gibson, D. G., Young, L., Chuang, R.-Y., Venter, J. C., Hutchison, C. A., and Smith, H. O. (2009) Enzymatic assembly of DNA molecules up to several hundred kilobases. Nat. Methods 6, 343-345.

(48) Pfefferkorn, J. A., Choi, C., Song, Y., Trivedi, B. K., Larsen, S. D., Askew, V., Dillon, L., Hanselman, J. C., Lin, Z., Lu, G., Robertson, A., Sekerke, C., Auerbach, B., Pavlovsky, A., Harris, M. S., Bainbridge, G., and Caspers, N. (2007) Design and synthesis of novel, conformationally restricted HMG-CoA reductase inhibitors. Bioorg. Med. Chem. Lett. 17, 45314537.

(49) Engler, C., Kandzia, R., and Marillonnet, S. (2008) A one pot, one step, precision cloning method with high throughput capability. PLoS One 3, e3647.

(50) Thompson, M. G., Sedaghatian, N., Barajas, J. F., Wehrs, M., Bailey, C. B., Kaplan, N., Hillson, N. J., Mukhopadhyay, A., and Keasling, J. D. (2018) Isolation and characterization of novel mutations in the pSC101 origin that increase copy number. Sci. Rep. 8, 1590.

(51) Batth, T. S., Singh, P., Ramakrishnan, V. R., Sousa, M. M. L., Chan, L. J. G., Tran, H. M., Luning, E. G., Pan, E. H. Y., Vuu, K. M., Keasling, J. D., Adams, P. D., and Petzold, C. J. (2014) A targeted proteomics toolkit for high-throughput absolute quantification of Escherichia coli proteins. Metab. Eng. 26, 48-56.

(52) Morrell, W. C., Birkel, G. W., Forrer, M., Lopez, T., Backman, T. W. H., Dussault, M., Petzold, C. J., Baidoo, E. E. K., Costello, Z., Ando, D., Alonso-Gutierrez, J., George, K. W., Mukhopadhyay, A., Vaino, I., Keasling, J. D., Adams, P. D., Hillson, N. J., and Garcia Martin, H. (2017) The Experiment Data Depot: A Web-Based Software Tool for Biological Experimental Data Storage, Sharing, and Visualization. ACS Synth. Biol. 6, 2248-2259. 